\title{
EVALUACION DE ACEITE E INSECTICIDAS PARA EL CONTROL DEL VIRUS DEL MOSAICO DORADO (VMDF) DEL FRIJOL EN CHIAPAS, MEXICO $^{1}$
}

\author{
Noé Becerra ${ }^{2}$, Guillermo Fraire ${ }^{2}$,Ernesto López ${ }^{3}$
}

\begin{abstract}
RESUMEN
Evaluación de aceite e insecticidas para el control del virus del mosaico dorado (VMDF) del frijol en Chiapas, México. Se evaluaron el efecto de dos aceites minerales y dos insecticidas sobre el control del VMDF en tres genotipos de frijol negro. Se encontró que en la variedad Negro Tacana dada su resistencia al virus pueden o no usarse los productos. En Negro Huasteco-81 pueden emplearse este tipo de productos para disminuir incidencia y aumentar rendimiento, desde 126 kilos con el tratamiento de Citrolina + Endosulfan hasta 320 kilos con los productos combinados de Imidacloprid 70 WS y 350 SC y de 281 kilos con Imidacloprid 70 WS. En Negro Cotaxtla-91 puede haber incrementos de 82 kilos con el tratamiento de Citrolina + Endosulfan.
\end{abstract}

\begin{abstract}
Evaluation of mineral oils and insecticides on the control of BGMV virus (Golden Mosaic) in Chiapas, Mexico. Upon evaluating the effect of two mineral oils and two insecticides on the control of the BGMV in three genotypes of black bean, it was found that the variety Negro Tacana given its resistance to the virus, they can or cannot use the products. In Negro Huasteco-81 we could employ this type of products, in order to disminish incidence and increase yield, from 126 kilos with the treatment of Citrolina + Endosulfan; upto 320 kilos with the combined products of Imidacloprid $70 \mathrm{WS}$ and $350 \mathrm{SC}$ and of 281 kilos with Imidacloprid 70 WS. In Negro Cotaxtla-91 can there be are increment of 82 kilos with the treatment of Citrolina + Endosulfan.
\end{abstract}

\section{INTRODUCCION}

La enfermedad del mosaico dorado del frijol (VMDF), transmitida por la mosquita blanca Bemisia tabaci, se presenta en el sureste de México causando severos daños. El manejo de la enfermedad se ha tratado de realizar por medio de aplicación de agroquímicos; sin embargo, el abuso de este tipo de productos puede traer como resultado que el insecto adquiera resistencia. Otro práctica que se ha implementado ha sido el uso de variedades resistentes, en este caso también el insecto puede romperla.

El uso de aceites minerales para el control de virosis se ha dado con frecuencia en varios cultivos principalmente en hortalizas (Simons, McLean y Kinsey, 1977 y Zitter y Ozaki, 1978); generalmente, se requieren concentraciones de al menos 2\%, para obtener una buena eficiencia (Zitter y Simons, 1980).

En trabajos de invernadero, las aspersiones de aceite han dado resultados en contra del Virus del Mosaico común y virus del amarillamiento del frijol (Walkley y Dance, 1979).

Otro cultivo donde se ha probado el uso de aceites es el tabaco, en contra del virus del jaspeado, usándose concentraciones de $1,1,5$ y $2 \%$ de citrolina, además de los aceites de maíz, olivo, cártamo y soya, siendo mejor el de citrolina al 2\% (Martínez, 1986).

Las aspersiones han sido efectivas en contra de los siguientes vectores Myzus persicae, Brevicoryne brassicae, Aphis fabae, A. gossypii, Macrosiphum

1 Presentado en la XLI Reunión Anual del PCCMCA en Honduras, América Central. 26 de marzo - 1 de abril, 1995.

2 Programa de Leguminosas Comestibles CIRGOC-INIFAP. C.E. "Cotaxtla” Apdo. Postal \#429. Veracruz, Ver. C.P. 91700. México.

3 Programa de Leguminosas Comestibles CIRPA-INIFAP. C.E. "Rosario Izapa". Apdo. Postal\# 51. Tapachula, Chiapas. C.P. 30700 México. 
euphorbiae, Rhopalosiphum maidis, Bemisia tabaci (Mattheus, 1970; Singh, Sastry y Sastry, 1973; Walkley y Dance, 1977; Ferro, Mackenzie y Margolies, 1980). El aceite puede también interferir con la transmisión de virus semipersistentes por áfidos y recientemente se ha reportado que la transmisión de los virus persistentes (como la enfermedad del amarillamiento del tomate), es reducida por el uso de aceites; la transmisión de virus semipersistentes, como el del enanismo clorótico del maíz por chicharritas y el virus del enrrollamiento de la hoja del tomate transmitido por la mosquita blanca, son factibles de controlarse (Zitter y Simons, 1980).

Como resultado de la aplicación de aceites se han obtenido reducciones de hasta ocho veces la diseminación de virus en el cultivo de chile en Florida; al compararse el promedio de infección en parcelas tratadas y no aplicadas da una relación de $15 \%$ en las primeras y $75 \%$ en las segundas (Zitter y Ozaki, 1978).

Los factores que afectan una buena eficiencia en las aspersiones de aceite son: 1) elección correcta de la combinación emulsificante-aceite; 2) concentración apropiada del aceite sin que cause daños al cultivo; 3) efectuar asperciones eficientes, para asegurar un excelente cubrimiento foliar; 4) no aplicar a temperaturas altas (arriba de $30^{\circ} \mathrm{C}$ ); 5) determinación de intervalos de aplicación según el cultivo, (de crecimiento rápido o lento); 6) determinar la especificidad del aceite; el de maíz previene la transmisión del Virus del Mosaico del betabel por M. persicae, pero no la del Amarillamiento del betabel; 7) el incremento de vectores puede disminuir la eficiencia del aceite; 8) la densidad de plantas influye en la tasa de incremento de la enfermedad y en los hábitos del vector; a mayor densidad se disminuye la diseminación; 9) los aceites no deben asperjarse sobre follaje húmedo, porque el cubrimiento puede ser pobre y la humedad puede redistribuir el aceite, causando pérdidas en la eficiencia (Simons y Zitter, 1980; Mattheus, 1970; Walker y Dance, 1979; Simons, McLean y Kinsey, 1977 y Zitter y Ozaki, 1978).

El producto Imidacloprid es un insecticida sistémico formulado principalmente para insectos chupadores; pertenece a un nuevo grupo de ingredientes activos las nitroguanidinas, el cual es de baja toxicidad para animales de sangre caliente y de una considerable actividad residual; la acción del producto esta basada en la interferencia de la transmisión de los impulsos en el sistema nervioso de los insectos (Bayer,1992).

El objetivo de este trabajo fue evaluar el uso de dos aceites minerales solos o combinados con insecti- cidas, sobre tres genotipos de frijol y probar un nuevo insecticida para disminuir la enfermedad del virus del mosaico dorado del frijol.

\section{MATERIALES Y METODOS}

Se establecieron dos experimentos, el primero el 20 de octubre de 1992, bajo condiciones de humedad residual. El diseño empleado fue de bloques al azar con seis repeticiones; la parcela experimental consistió de cuatro surcos de cinco metros de largo, separados a 50 $\mathrm{cm}$; la fertilización se efectuó aplicando la fórmula 40-30-00. Se usaron las Variedades Negro Huasteco-81 y Negro Cotaxtla-91. Los tratamientos fueron los siguientes para cada una de las variedades:

1.- Aceite citrolina 2\%

2.- Aceite citrolina $2 \%+$ Endosulfan

3.- Aceite mineral Saf-t-Add $2 \%$

4.- Aceite mineral Saf-t-Add 2\% + Endosulfan

5.- Testigo sin aplicación

Las fechas de aplicación de los tratamientos fueron a los 22, 29 y 36 días después de sembrado, utilizando una bomba de motor Robin. Los parámetros para evaluar los tratamientos fueron: a) evaluaciones de incidencia de plantas enfermas a los 20, 28, 35 y 41 días de sembrado; ésta consistía en contar el número de plantas enfermas con virus en cada uno de los surcos de los tratamientos y repeticiones, posteriormente se obtenía el porcentaje de plantas enfermas. Al final del experimento se evaluó el rendimiento de los dos surcos centrales ajustando la humedad del grano a 14\%. Con los datos se realizaron análisis de varianza y pruebas de Duncan al 0,05, para comparación de medias en aquellas variables que tenían significancia.

Además de lo anterior, se marcaron plantas que mostraban los inicios de síntomas de mosaico en cuatro diferentes fechas $(28,35,40$ y 48 días después de sembrado el cultivo), para evaluar la pérdida en rendimiento en cada una de ellas.

El segundo experimento se estableció bajo las mismas condiciones que el anterior, la fecha de siembra fue el 11 de octubre de 1993. El diseño empleado fue un factorial en bloques al azar con seis repeticiones, la parcela experimental consistió de cuatro surcos de 5,0 de largo separados a $50 \mathrm{~cm}$; la fertilización se efectuó aplicando la fórmula 40-40-00 kg/ha de $\mathrm{N} \mathrm{P}_{2} \mathrm{O}_{5}$ y $\mathrm{K}_{2} 0$, respectivamente; a los 30 días se complementó con una fertilización foliar de urea (1 kilogramo en 20 litros de agua). 
Se usaron las variedades Negro Huasteco-81 y Negro Tacana; los tratamientos fueron los siguientes, para cada uno de los genotipos:

1.- Aceite Saf-t-Add $2 \%$

2.- Aceite Saf-t-Add 2\% + Endosulfan

3.- Endosulfan

4.- Imidacloprid $70 \mathrm{WS}$

5.- Imidacloprid $350 \mathrm{SC}$

6.- Imidacloprid $70 \mathrm{WS}+$ Imidacloprid $350 \mathrm{SC}$

7.- Testigo

Las fechas de aplicación de los tratamientos fueron cada siete días en los tratamientos 1, 2, 3 (cuatro aplicaciones); en el tratamiento 4 fue a la siembra impregnando la semilla; el tratamiento 5 se efectuó a los siete y 26 días de sembrado; el tratamiento 6 fue a la siembra y a los 26 días, respectivamente, utilizando para las aspersiones foliares una bomba de motor Robin. Las dosis aplicadas fueron de 20 mililitros de aceite por litro de agua: Endosulfan 2 mililitros por litro de agua: Imidacloprid 70 WS 4 gramos por kilo de semilla e Imidacloprid 2 mililitros por litro de agua; al testigo se le aplicó solamente agua. Los parámetros para evaluar los tratamientos fueron una evaluación de incidencia de plantas con síntomas de virus a los 57 días de sembrado, mediante una escala de nueve grados donde:

$1=$ síntomas ausentes e incidencia $0 \%$

$2=$ síntomas dudosos e incidencia $1-10 \%$

$3=$ síntomas débiles e incidencia $11-25 \%$

$4=$ síntomas moderados e incidencia $26-40 \%$
$5=$ síntomas intermedios e incidencia $41-60 \%$
$6=$ síntomas generales e incidencia $61-75 \%$
$7=$ síntomas intensos e incidencia $76-90 \%$
$8=$ síntomas severos e incidencia $91-99 \%$
$9=$ muerte e incidencia de $100 \%$

Al final se evaluaron el número de plantas cosechadas y el rendimiento en $\mathrm{kg} / \mathrm{ha}$ ajustado al $14 \%$ de humedad; con los datos se hicieron análisis de varianza y prueba de Duncan al 0,05.

\section{RESULTADOS Y DISCUSION}

\section{Primer experimento ciclo OI-1992-93}

Al efectuar el análisis de varianza para la variable incidencia se detectaron diferencias altamente significativas y la prueba de Duncan indicó que los tratamientos testigos de las dos variedades mostraron los porcentajes más altos; en tanto que los tratamientos de Aceite Saf-t-Add + Endosulfan, en las dos variedades, presentaban las calificaciones más bajas de todas, los demás tratamientos fueron intermedios (Cuadro 1).

Se encontró para rendimiento diferencias altamente significativas, los tratamientos con los rendimientos más altos fueron: Citrolina + Endosulfan, en la variedad Negro Huasteco-81; Aceite Saf-t-Add + Endosulfan y Saf-t-Add solo en la misma variedad. En la variedad Negro Cotaxtla-91 el mejor tratamiento fue el de Aceite Saf-t-Add + Endosulfan; todos los demás tratamientos fueron iguales para esta variedad (Cuadro 1). Estos

Cuadro 1. Incidencia del mosaico dorado y rendimiento por tratamiento (aceites e insecticida), en el Estado de Chiapas. Ciclo Otoño-Invierno. 1992-93.

\begin{tabular}{llcc}
\hline \multirow{2}{*}{ Variedad } & Tratamiento & \multicolumn{2}{c}{ Prueba de Duncan } \\
\cline { 3 - 4 } & & Rendimiento kg/ha & Incidencia \% \\
\hline N.H.-81 & Citrolinalina + Endosulfan & $260,00 \mathrm{a}$ & $5,90 \mathrm{bc}$ \\
N.H.-81 & Saf-t-Add + Endosulfan & $241,67 \mathrm{a}$ & $4,63 \mathrm{c}$ \\
N.H.-81 & Saf-t-Add & $223,83 \mathrm{a}$ & $7,93 \mathrm{abc}$ \\
N.H.-81 & Citrolinalina & $193,33 \mathrm{ab}$ & $8,48 \mathrm{ab}$ \\
N.H.-81 & Testigo & $134,33 \mathrm{bc}$ & $10,35 \mathrm{a}$ \\
N.C.-91 & Saf-t-Add + Endosulfan & $128,50 \mathrm{bc}$ & $4,42 \mathrm{c}$ \\
N.C.-91 & Citrolina. + Endosulfan & $100,50 \mathrm{c}$ & $5,20 \mathrm{bc}$ \\
N.C.-91 & Saf-t-Add & $83,83 \mathrm{c}$ & $7,63 \mathrm{abc}$ \\
N.c.-91 & Citrolinalina & $81,17 \mathrm{c}$ & $7,45 \mathrm{abc}$ \\
N.C.-91 & Testigo & $46,00 \mathrm{c}$ & $10,30 \mathrm{a}$ \\
\hline
\end{tabular}

SIGNIFICANCIA ANDEVA 
resultados concuerdan con los de Singh, Sastry y Sastry (1973), ya que dichos investigadores al utilizar aspersiones de aceites al $0,37 \%, 0,74 \%$ y $1,11 \%$,para el control del Virus del enrrollamiento de la hoja del tomate, encontraron que el rendimiento en parcelas tratadas era de 75,06, 89,00 y 93,53 quintales por hectárea $(\mathrm{q} / \mathrm{ha})$, respectivamente, mientras que en los controles no asperjados la producción fue de 44,88 q/ha.

Zitter y Ozaki (1978), en el cultivo de pepino, encontraron diferencias significativas, al comparar promedios de infección de virus en parcelas no tratadas con aceite (33\%), en tanto que las asperjadas con aceite JMS style fueron de (4\%); la producción también fue significativa ya que se obtuvieron 105 libras en las no tratadas y 288 en las asperjadas.

En el presente experimento la baja cantidad de rendimiento estuvo influenciada, además de la virosis, por una falta de humedad en las etapas de floración y llenado de vainas, aunado a la presencia de pudriciones radiculares causadas por los hongos Sclerotium rolfsii y Rhizoctonia solani; aún con todo esto, los análisis pudieron detectar diferencias entre los tratamientos.

En ambas variedades los tratamientos donde se mezclaron aceite e insecticida se comportaron mejor siendo quizás este último el responsable del incremento de producción, ya que los tratamientos individuales de aceites tuvieron menor rendimiento.

En la Figura 1 se muestra la incidencia del VMDF que se presentó por muestreo en cada uno de los tratamientos en la variedad Negro Huasteco-81; puede observarse que los tratamientos de Aceite Saf-t-Add + Endosulfan presenta la menor incidencia. En la variedad Negro Cotaxtla-91 también se repite esto y para esta variedad en particular también funcionó la Citrolina +
Endosulfan (Figura 2). Estos resultados concuerdan con los de Ferro, Mackenzie y Margolies (1980), ya que en el cultivo de maíz al asperjar aceite mineral JMS stylete el porcentaje de plantas infectadas con el Virus del Mosaico enanismo del maíz fue de 15,4; las de aceite y el aficida Aldicarb promediaron 16,7, las parcelas con solo el aficida tuvieron 27,7 , en tanto que los controles promediaron 23,2 .

De las plantas que se marcaron en las diferentes fechas para evaluar las pérdidas en producción al final del ciclo, ninguna llego a producción ya que el $100 \%$ se murió o simplemente se enchinaron sin llegar a producir vainas. Estos resultados concuerdan con los de Gálvez y Morales (1989), quienes mencionan que el virus puede provocar pérdidas hasta del $100 \%$, bajo condiciones de alta incidencia.

\section{Segundo experimento ciclo OI- 1993-94}

Se encontraron diferencias altamente significativas para la variedad, el producto y la interacción con la incidencia, (Cuadro 2); el aceite y el testigo presentan los valores más altos, en cambio el Endosulfan y el Imidacloprid combinando las dos formulaciones da el más bajo, siendo los restantes intermedios; en el caso de rendimiento, no se detectaron diferencias significativas para variedad y producto pero si para la interacción, siendo solamente significativas; aunque el análisis de medias no mostró grupos, se puede observar diferencias de hasta 230 kilos, entre la combinación de Imidacloprid y el tratamiento de aceite y de 186 entre el mismo tratamiento y el testigo (Cuadro 2). Lo anterior concuerda con Bayer (1992), quien menciona que el producto funciona bien para insectos chupadores, como en este caso la mosquita blanca, ya que los tratamientos donde se aplicó Imidacloprid, en sus dos formulaciones, tuvieron la incidencia más baja de virosis.

Cuadro 2. Incidencia de mosaico dorado y rendimiento por producto (aceite e insecticidas) en el Estado de Chiapas. Ciclo Otoño-Invierno. 1993-94.

\begin{tabular}{lccc}
\hline Producto & $\begin{array}{c}\text { Incidencia } \\
(\boldsymbol{\%})\end{array}$ & Rendimiento (kg/ha) & $\begin{array}{c}\text { Diferencia vs } \\
\text { mejor (kg/ha) }\end{array}$ \\
\hline Aceite 3,75 a 598,92 a 230,16 & & & \\
Testigo & $3,67 \mathrm{a}$ & $643,17 \mathrm{a}$ & 185,91 \\
Imida 70 WS & $3,58 \mathrm{ab}$ & $813,25 \mathrm{a}$ & 15,83 \\
Imida 350 SC & $3,08 \mathrm{abc}$ & $708,75 \mathrm{a}$ & 120,33 \\
Aceite + Endo. & $2,92 \mathrm{abc}$ & $666,67 \mathrm{a}$ & 162,42 \\
Endosulfan & $2,75 \mathrm{bc}$ & $820,83 \mathrm{a}$ & 8,25 \\
Imida 70 + 350 & $2,67 \mathrm{c}$ & $829,08 \mathrm{a}$ & 0,00 \\
\hline
\end{tabular}




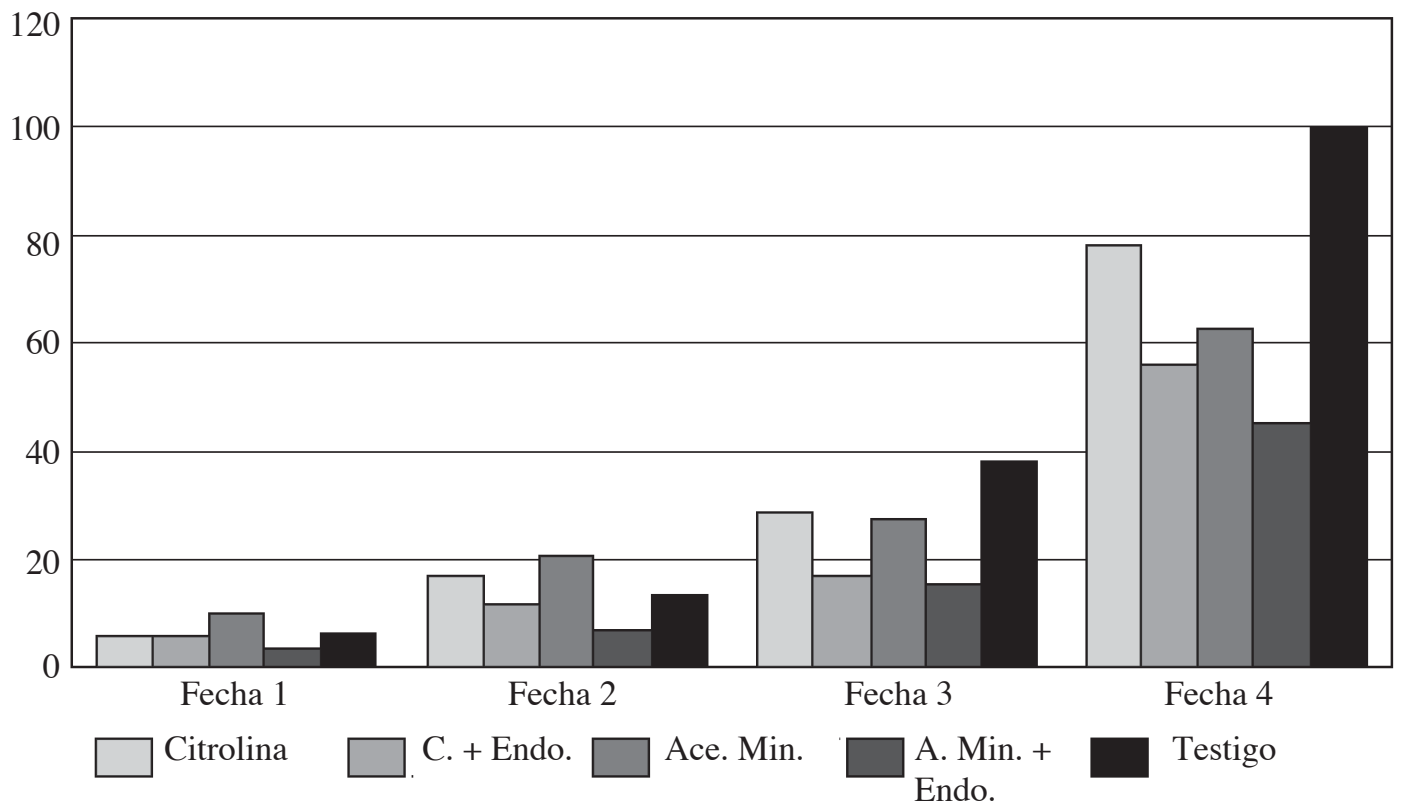

Fig. 1. Efecto de aceites e insecticidas sobre la incidencia del VMDF en la variedad Negro Huasteco-81. Chiapas, México. Ciclo O-I. 1992-1993.

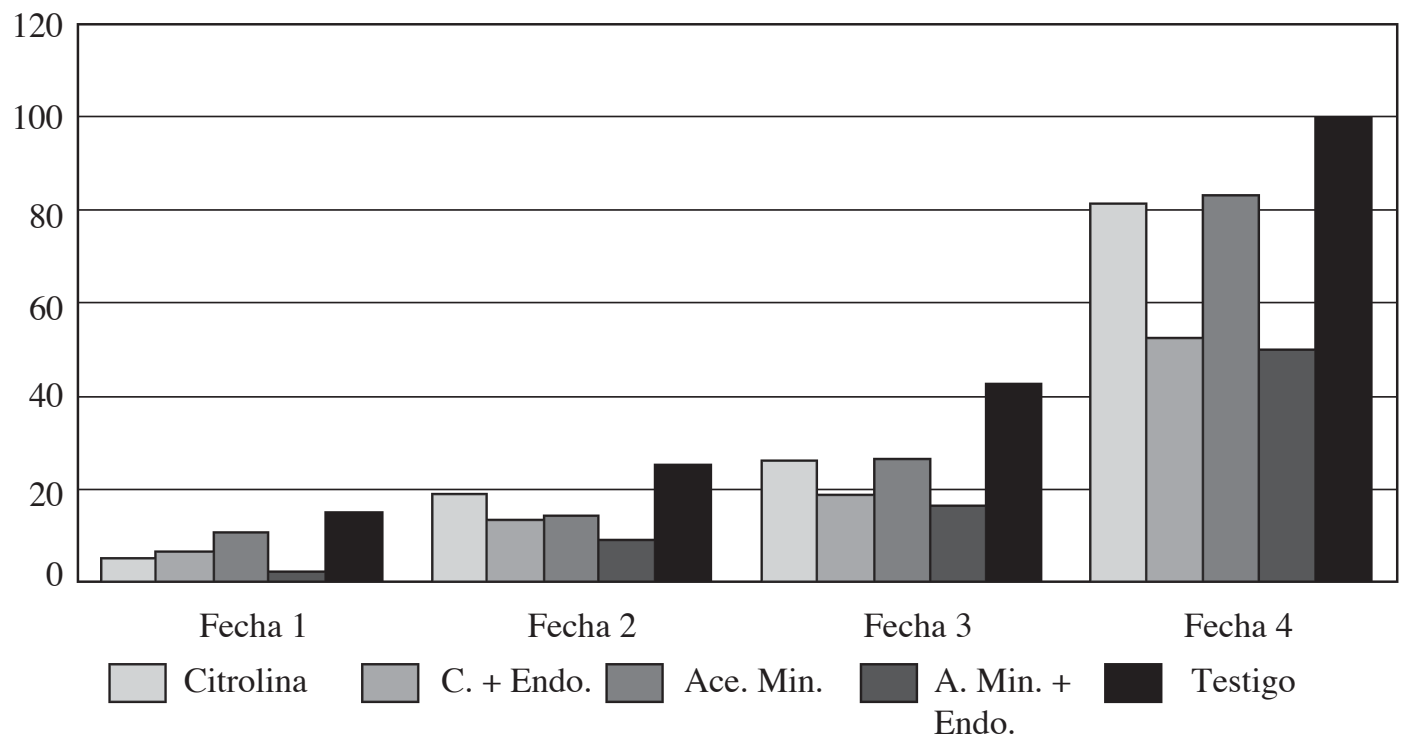

Fig. 2. Efecto de aceites e insecticidas sobre la incidencia del VMDF en la variedad Negro Cotaxtla91. Chiapas, México. Ciclo O-I. 1992-1993. 
Al desglosar el efecto del producto sobre la incidencia el análisis de medias se detectó que la variedad Negro Tacana, independientemente del producto aplicado, obtuvo las incidencias más bajas; en tanto los mejores tratamientos en la variedad Negro Huasteco-81 fueron la combinación del Imidacloprid, el Endosulfan solo y combinado con aceite y las formulaciones del Imidacloprid individuales, el testigo y el aceite solo, tuvieron los valores más altos (Cuadro 3).

Al desglosar el efecto del producto sobre variedad y rendimiento, mediante la prueba de medias de Duncan (Cuadro 3), se observaron los rendimientos más altos en los tratamiento de Imidacloprid 350 SC y Aceite + Endosulfan en la variedad Negro Tacana. Otro grupo se formó con la mayoría de los tratamientos en ambas variedades y el último lugar con el rendimiento más bajo fue el de Aceite + Endosulfan y aceite solo en la variedad Negro Huasteco-81.

Es importante mencionar que la incidencia del VMDF fue baja y que la variedad Negro Tacana, tiene mayor tolerancia y/o resistencia al VMDF que Negro Huasteco-81; esto corrobora los resultados obtenidos por esta variedad en años anteriores (Fraire, 1993); además, con y sin protección, tiene buen rendimiento; sin embargo, la aplicación de Imidacloprid 350 SC o aceite mineral Saf-t-Add + Endosulfan puede incrementar su rendimiento en 171 y $156 \mathrm{~kg} / \mathrm{ha}$.

En la variedad Negro Huasteco-81 los mejores rendimientos correspondieron a los tratamientos de Imidacloprid $70 \mathrm{WS}+350 \mathrm{SC}$ e Imidacloprid $70 \mathrm{WS}$.

\section{LITERATURA CITADA}

BAYER, 1992. Confidor. Technical Information. In: Japan and United States. p 25.

FERRO, D.N.; MACKENZIE, J.D.; MARGOLIES, D.C. 1980. Effect of mineral oil and a systemic insecticide on field spread of aphid-borne Maize Dwarf Mosaic Virus in sweet corn. Journal of Economic Entomology. 73: $730-735$.

FRAIRE, V.G. 1993. Negro Tacana, una nueva variedad de frijol para la Costa de Chiapas. Folleto técnico No. 5. CERI-CRPS-INIFAP. Tapachula, Chiapas.

Cuadro 3. Porcentaje de incidencia de visosis y rendimiento sobre dos genotipos de frijol en un experimento de aceite e insecticidas en el Estado de Chiapas. Ciclo Otoño-Invierno. 1993-94. CECOT-CIRGOC-INIFAP-SARH.

\begin{tabular}{|c|c|c|c|}
\hline \multirow[t]{2}{*}{ Genotipos } & \multirow[t]{2}{*}{ Producto } & \multicolumn{2}{|c|}{ Prueba de Duncan 1} \\
\hline & & \% Incidencia & Rendimiento (kg/ha) \\
\hline N.H.-81 & Testigo & $5,67 \mathrm{~A}$ & $546,17 \mathrm{abc}$ \\
\hline N.H.-81 & Aceite $2 \%$ & $5,50 \mathrm{ab}$ & $428,67 \mathrm{c}$ \\
\hline N.H.-81 & Imida $70 \mathrm{WS}$ & $5,33 \mathrm{abc}$ & $827,67 \mathrm{abc}$ \\
\hline N.H.-81 & Imida $350 \mathrm{Sc}$ & 4,17 abcd & $506,33 \mathrm{abc}$ \\
\hline N.H.-81 & Aceite + Endo & $4,00 \mathrm{bcd}$ & $437,33 \mathrm{bc}$ \\
\hline N.H.-81 & Endosulfan & $3,83 \mathrm{~cd}$ & $766,83 \mathrm{abc}$ \\
\hline N.H.-81 & Imida $70+350$ & 3,50 de & $866,67 \mathrm{abc}$ \\
\hline N. Tacaná & Aceite $2 \%$ & $2,00 \quad \mathrm{f}$ & $769,17 \mathrm{abc}$ \\
\hline N. Tacaná & Imida $350 \mathrm{SC}$ & 2,00 & 911,17 a \\
\hline N. Tacaná & Imida $70+350$ & 1,83 & $791,50 \mathrm{abc}$ \\
\hline N. Tacaná & Imida $70 \mathrm{WS}$ & 1,83 & $798,83 \mathrm{abc}$ \\
\hline N. Tacaná & Aceite + Endo. & 1,83 & $896,00 \mathrm{ab}$ \\
\hline N. Tacaná & Endosulfan & $1,67 \quad \mathrm{f}$ & $874,83 \mathrm{abc}$ \\
\hline N. Tacaná & Testigo & $1,67 \mathrm{f}$ & $740,17 \mathrm{abc}$ \\
\hline
\end{tabular}

Imida $=$ imidacloprid $\quad$ N.S. $=$ No Significativa

Endo $=$ Endosulfan

$*$ Significancia al $0,05 \%$.

$* *=$ Significancia al $0,01 \%$.

$1=$ Tratamientos con la misma letra son estadisticamente iguales 
GALVEZ, G.E.; MORALES, F.J. 1989. White-fly transmitted viruses. In: Bean production problems in the tropics. Second Ed. Schwartz H.F. and Pastor Corrales M.A. (Eds.). CIAT. Colombia. pp. 379-406.

MARTÍNEZ, J.L. 1986. Memoria del XII Congreso Nacional de Fitopatología. 22-25 julio Tuxtla Gutierrez, Chiapas. p. 51

MATTHEUS, F.R.E. 1970. Plant Virology. Academic Press. N.Y., Sn. Francisco and London. p. 778.

SIMONS, N.J.; MCLEAN, L.D.; KINSEY, G.M. 1977. Effects of mineral oil on probing behavior and transmission of stylet-borne viruses by Myzus persicae. Journal of Economic Entomology. 70: 309-315.
SINGH, S.J.; SASTRY, S.M.; SASTRY, K.S. 1973. effect of oil spray on the control of Tomato Leaf-Curl Virus in field. Indian J. Agric. Sci. 43: 669-672.

WALKLEY, A.D.G.; DANCE C.M. 1979. The effect of oil sprays on aphid transmission of Turnip Mosaic, Beet Yellows, Bean Common and Bean Yellow Mosaic Viruses. Plant Disease Reporter. 63: 877-881.

ZITTER, A.I.; OZAKI, H.Y. 1978. Aphid-borne vegetable viruses controlled with oil sprays. Proc. Fla. State Hort. Soc. 91: 287-289.

ZITTER, A.I.; SIMONS N.J. 1980. Management of viruses by alteration of vector efficiency and by cultural practices. Ann. Rev. Phytopathology. 18: 289-310. 